\title{
DEALING WITH OUTLIERS AND OFFSETS IN RADIOCARBON DATING
}

\author{
Christopher Bronk Ramsey \\ Research Laboratory for Archaeology, University of Oxford, Dyson Perrins Building, South Parks Road, Oxford, OX1 3QY, \\ United Kingdom. Email: christopher.ramsey@ rlaha.ox.ac.uk.
}

\begin{abstract}
The wide availability of precise radiocarbon dates has allowed researchers in a number of disciplines to address chronological questions at a resolution which was not possible 10 or 20 years ago. The use of Bayesian statistics for the analysis of groups of dates is becoming a common way to integrate all of the ${ }^{14} \mathrm{C}$ evidence together. However, the models most often used make a number of assumptions that may not always be appropriate. In particular, there is an assumption that all of the ${ }^{14} \mathrm{C}$ measurements are correct in their context and that the original ${ }^{14} \mathrm{C}$ concentration of the sample is properly represented by the calibration curve.
\end{abstract}

In practice, in any analysis of dates some are usually rejected as obvious outliers. However, there are Bayesian statistical methods which can be used to perform this rejection in a more objective way (Christen 1994b), but these are not often used. This paper discusses the underlying statistics and application of these methods, and extensions of them, as they are implemented in OxCal v 4.1. New methods are presented for the treatment of outliers, where the problems lie principally with the context rather than the ${ }^{14} \mathrm{C}$ measurement. There is also a full treatment of outlier analysis for samples that are all of the same age, which takes account of the uncertainty in the calibration curve. All of these Bayesian approaches can be used either for outlier detection and rejection or in a model averaging approach where dates most likely to be outliers are downweighted.

Another important subject is the consistent treatment of correlated uncertainties between a set of measurements and the calibration curve. This has already been discussed by Jones and Nicholls (2001) in the case of marine reservoir offsets. In this paper, the use of a similar approach for other kinds of correlated offset (such as overall measurement bias or regional offsets in the calibration curve) is discussed and the implementation of these methods in OxCal v 4.0 is presented.

\section{INTRODUCTION}

Before considering statistical methods for the treatment of outliers and offsets, it is important to understand the underlying mechanisms and issues. There are essentially 4 main reasons why in any context radiocarbon dates might not give the "right" result:

- The ${ }^{14} \mathrm{C}$ measurement of a particular sample might not be correct (s).

- The ${ }^{14} \mathrm{C}$ ratio of a sample might be different from that of the associated reservoir (r).

- A whole set of ${ }^{14} \mathrm{C}$ measurements might be biased in some way relative to the calibration curve- either because the measurements themselves are biased or because the reservoir from which the sample draws its carbon might not have the expected ${ }^{14} \mathrm{C}$ isotope ratio (d).

- The sample measured might not relate to the timing of the event being dated ( $\mathrm{t}$ ).

Ideally, the uncertainty quoted in the ${ }^{14} \mathrm{C}$ measurement covers the first possibility (s), though in some instances it may be that the errors in the measurements are not normally distributed. For this reason, it might be that in ${ }^{14} \mathrm{C}$ calibration, rather than adopting a Normal distribution, for a more robust model we should be using a longer-tailed distribution (such as a Student's $t$ distribution). Another approach has been suggested where we assume that in a small proportion of cases the measurement effectively has an uncertainty, which is larger by some factor (Christen 1994b, 2003).

In the second case (r), the measurement is correct, but the ${ }^{14} \mathrm{C}$ isotope ratio might be different from that of the calibration curve at the associated age for some reason. This might be due to short-term fluctuations in ${ }^{14} \mathrm{C}$ concentrations in particular reservoirs or due to an admixture of carbon from different sources. Such offsets are analogous to the first category but will not be related in any way to the measurement uncertainty or be improved by multiple measurement.

The 2 categories given for the third reason (d) are very different in their cause, but essentially the same in their effect. The situation here is that the measurements made for the calibration curve and 
those for the sample have a systematic offset relative to one another. Where such offsets are recognized, they can be taken into account using a $\Delta R$ correction (Stuiver and Braziunas 1993). In principle, unknown offsets can be treated in similar way using a $\Delta \mathrm{R}$ with a mean of zero and an uncertainty that reflects the possible scale of offsets between the measurement sets. The correct statistical treatment of such systematic offsets has been described by Jones and Nicholls (2001).

Finally, we come to what is probably the most common form of outlier, where the sample does not for some reason relate to the dated event in the intended way (t). Here, the ${ }^{14} \mathrm{C}$ measurements are correct and the values relate correctly to the calibration curve data sets, but there is some sort of calendar offset between the measurement and the event of interest. In some instances, such outliers are due to aspects of the deposition process that are hard to understand. In other cases, we know why samples might be (or are) outliers: for example, in the case of charcoal we usually expect the samples to be older than their context. This type of outlier is not restricted specifically to ${ }^{14} \mathrm{C}$ dating or contamination at the sampling stage.

All of these types of outlier can be treated statistically using essentially similar methods but in slightly different ways. The purpose of this paper is to summarize these approaches and describe the implementation of their algorithms in v 4.1 of the analysis program OxCal (Bronk Ramsey 1995, 2001, 2008).

\section{TREATMENT OF OUTLIERS}

In general, there are two main ways of dealing with outliers. The first is to try to identify all outliers and then eliminate them manually from the analysis. If this is possible, then it is probably the best approach since it is then entirely clear what data are being used to support the analysis. The other approach is to assume that we can never really be sure whether any particular measurement is an outlier, but to weight samples according to how likely they are to be correct in a model averaging approach. This outlier analysis approach requires us to provide a prior probability for how likely any individual measurement is to be wrong and then some model to determine how we should revise this in the context of all of the other information available.

\section{Manual Rejection}

How you identify outliers for rejection is a complex topic. The most important considerations are the sample context and details of the measurement process itself. These should allow us to identify which samples might have give anomalous ${ }^{14} \mathrm{C}$ measurements or have a complex depositional history. It is also possible to use statistical methods to indicate which samples seem anomalous within their context to support these decisions. You can either use the outlier analysis methods outlined by Christen (1994b) and in this paper or use the agreement index (Bronk Ramsey 1995) calculated by OxCal. In practice, for identification purposes both methods work well-indeed, in almost all instances the same samples will be identified by either. In both cases, the level at which we start to reject samples is somewhat arbitrary. If you use the agreement index method, unless a sample has been rejected, all measurements are given equal weight. With outlier analysis samples are progressively down-weighted as they are more likely to be outliers and so the results from the analysis are essentially an average between a model in which the measurement is accepted and one in which it is rejected. If you do not wish to have model averaging, but do wish to use outlier analysis solely for outlier detection, you should first run a model with outlier analysis, see which measurements are likely to be spurious and then run it again, without outlier analysis but with some of the spurious results removed entirely. 
In OxCal v 4.1, there are 3 tools that can be used to help with the manual elimination of outliers. The first is the calculation of the agreement index for each sample; if this falls below $60 \%$, rejection should be considered. However, it should be remembered that approximately 1 in 20 samples are likely to fall below this level and such rejection should also be based on other criteria. Secondly, an overall agreement index is calculated $A_{\text {model }}$ and if this is above $60 \%$ it probably indicates that there is no problem with the model as a whole (and therefore no samples need be rejected). Finally, there is a command Outlier(), which can be used to flag a measurement as a definite outlier and remove it from the model (note in earlier versions of OxCal this command was Question() but otherwise worked in the same way).

\section{Outlier Analysis}

In order to deal with outliers statistically, we need to have some sort of a model for how we expect them to be distributed. We also ought to define, in the case of ${ }^{14} \mathrm{C}$, whether we think it is the ${ }^{14} \mathrm{C}$ measurement that is incorrect for some reason or if it is the context that is uncertain. Usually in ${ }^{14} \mathrm{C}$ dating, we assume that a specific ${ }^{14} \mathrm{C}$ measurement $r_{i}$ will differ from the prevailing ${ }^{14} \mathrm{C}$ concentration $r\left(t_{i}\right)$, given to us by the calibration curve, by an amount $\varepsilon_{i}$ such that:

$$
\begin{gathered}
r_{i}=r\left(t_{i}\right)+\varepsilon_{i} \\
\varepsilon_{i} \sim N\left(0, s_{i}^{2}+\left(s\left(t_{i}\right)\right)^{2}\right)
\end{gathered}
$$

So that the difference is entirely accounted for by the uncertainties quoted for the measurement $\left(s_{i}\right)$ and the calibration curve $\left(s\left(t_{i}\right)\right)$. In outlier analysis, we need to be able to deal with other kinds of offset. We introduce another parameter $\phi_{i}$, which is 1 if the sample is an outlier and 0 if it is not.

OxCal v 4.1 provides the tools to set up such models. The tools are generic and allow a wide variety of models to be employed. However, it should be stressed that it is usually best to keep things fairly simple and in most cases one model should be all that is required. The two commands that have been introduced to provide outlier analysis are:

Outlier_Model([name,] distribution [,scale [,type] ] \}) ;

Outlier ([name, $]$ [prior $])$;

The Outlier_Model () command defines the model and the Outlier() command allows it to be applied to specific ${ }^{14} \mathrm{C}$ dates or other likelihood information in the model. The parameters of the model are:

- name - this is the name of the model; this can be used to allow the specification of more than one outlier model; if the name is not specified, the last model defined will be used for any outlier analysis. If for example you wish to use a special model for all charcoal samples, the name "charcoal" can be given to both the Outlier_Model () and the associated Outlier() commands.

- distribution - this defines how the outliers are to be distributed (distribution $D_{1}$ ); examples of useful distributions are $T(5)$, a Student's $t$ distribution with 5 degrees of freedom; $N(0,1)$, a simple normal distribution; or $\operatorname{Exp}(1,-10,0)$, an exponential distribution with an exponential constant $\tau$ of 1 taken over the range -10 to 0 .

- scale - this defines the scaling of the outliers, expressed in powers of 10. This can be a single number such as 0 for no scaling or 2 for a scale of $100 \mathrm{yr}$; it can also be a distribution (distribution $\left.D_{2}\right)$ such as $U(0,4)$ for a scale of anywhere between $1-10,000 \mathrm{yr}$. In this case, the analysis will determine the appropriate scale. 
- type - this defines the kind of outlier you have; the options are " $t$ " for outliers in the time variable, " $r$ " for those in the ${ }^{14} \mathrm{C}$ isotope ratio, and " $\mathrm{s}$ " for those that scale with the uncertainty in the ${ }^{14} \mathrm{C}$ concentration.

- prior - for any specific measurement this defines the prior probability that the sample is an outlier; a typical value for this would be 0.05 for a 1 in 20 chance that the measurement needs to be shifted in some way. The posterior probability for the measurement being an outlier will be determined by the analysis.

The distribution and scale parameter can be defined in a number of ways in OxCal. They can either be constant numbers (this only makes sense for the scale) or they can be distributions. The relevant distributions defined in OxCal are shown in Table 1.

Table 1 Distribution definitions in OxCal; the optional resolution parameter defines the bin size during the MCMC analysis; if not specified, a suitable default is chosen.

\begin{tabular}{|c|c|c|}
\hline Definition & Example & Meaning \\
\hline $\operatorname{Exp}([$ name,$]$ tau, from, to $[$,resolution $])$ & $\operatorname{Exp}(1,-10,0)$ & $\begin{array}{l}\text { Exponential distribution range } \\
-10-0 \text { with } \tau=1\end{array}$ \\
\hline $\mathrm{N}([$ name,$]$ mu, sigma [,resolution $]]) ;$ & $N(0,2)$ & Normal distribution $\mu=0, \sigma=2$ \\
\hline $\mathrm{T}([$ name, freedom, $[$ scale $[$,resolution $]]) ;$ & $T(5)$ & $\begin{array}{l}\text { Student's } t \text { distribution } 5 \text { degrees } \\
\text { of freedom }\end{array}$ \\
\hline $\mathrm{U}([$ name,$]$ from to $[$,resolution $]) ;$ & $U(0,1)$ & Uniform distribution range $0-1$ \\
\hline
\end{tabular}

To see how these commands are to be used in practice, we will look at some specific applications. You can also see the examples in the following section.

First of all, we will consider the situation where the ${ }^{14} \mathrm{C}$ measurement itself might be at fault. We will further assume that any offsets are in proportion to the uncertainty quoted in the date. In this situation, the model outlined by Christen (2003) is most appropriate. In this model (s-type), any shift in the measurement is drawn from a normal distribution, which has double the uncertainty of the measurement:

$$
\begin{aligned}
r_{i} & =r\left(t_{i}\right)+\varepsilon_{i}+\phi_{i} \delta_{i} s_{i} \\
\varepsilon_{i} & \sim N\left(0, s_{i}^{2}+\left(s\left(t_{i}\right)\right)^{2}\right) \\
\phi_{i} & \sim \operatorname{Bernoulli}\left(q_{i}\right) \\
\delta_{i} & \sim N\left(0,2^{2}\right)
\end{aligned}
$$

where $q_{i}$ is the prior probability that the sample is an outlier. In OxCal, this can be specified by:

Outlier_Model ("SSimple",N(0,2), 0, "s");

Effectively, this model draws the shifts from a normal distribution with a mean of zero and a standard deviation of 2 and they are then multiplied by the uncertainty in the date and applied to the ${ }^{14} \mathrm{C}$ measurement. So, if for example the uncertainties in all the measurements are 50, the possible shifts are drawn from a normal distribution with a mean of zero and a standard deviation of 100. This is the default model for ${ }^{14} \mathrm{C}$ dates if no other is specified.

Supposing instead we have some other reason why the ${ }^{14} \mathrm{C}$ dates and those in the calibration curve may not be the same-perhaps there is possible contamination, or addition of ${ }^{14} \mathrm{C}$ from other reser- 
voirs. In such cases (r-type), the offsets will not be related to the uncertainty in the measurement. In these cases, our outlier model is modified:

$$
\begin{aligned}
r_{i} & =r\left(t_{i}\right)+\varepsilon_{i}+\phi_{i} 10^{u} \delta_{i} \\
\varepsilon_{i} & \sim N\left(0, s_{i}^{2}+\left(s\left(t_{i}\right)\right)^{2}\right) \\
\phi_{i} & \sim \operatorname{Bernoulli}\left(q_{i}\right) \\
\delta_{i} & \sim D_{1} \\
u & \sim D_{2}
\end{aligned}
$$

where $u$ is a scaling parameter common to the model as a whole and where $D_{1}$ and $D_{2}$ are distributions we can choose, according to modeling preference, from those given in Table 1. We might know the scale of such offsets, in which case we can fix $u$. If we know that they are likely to be of the order of a hundred years, we can let them be drawn from a normal distribution with a mean of zero and a standard deviation of 100 , by setting $u=0$ and $D_{1}$ to $N\left(0,100^{2}\right)$ :

Outlier_Model ("RSimple",N (0,100),0,"r");

If we do not know what sort of offset we are expecting, we can allow the model to find the scale (anywhere between $10^{0}$ and $10^{4}$ ) and so use $U(0,4)$ for $D_{2}$ and let the possible shifts be drawn from a longer-tailed Student $t$ distribution by using $T(5)$ for the distribution $D_{2}$ instead:

Outlier_Model ("RScaled", T (5), U (0,4), "r") ;

The case where there might be a systematic offset between the measurements and the calibration curve (d-type) is a special case and is discussed in the section "Systematic Offsets Relative to the Calibration Curve."

In many cases, though, the possible offsets are not in the ${ }^{14} \mathrm{C}$ scale but in the time scale (t-type). This type of outlier is applicable to other dating methods as well as ${ }^{14} \mathrm{C}$. In the ${ }^{14} \mathrm{C}$ case, we would then define:

$$
\begin{aligned}
r_{i} & =r\left(t_{i}+\phi_{i} 10^{u} \delta_{i}\right)+\varepsilon_{i} \\
\varepsilon_{i} & \sim N\left(0, s_{i}^{2}+\left(s\left(t_{i}+\phi_{i} 10^{u} \delta_{i}\right)\right)^{2}\right) \\
\phi_{i} & \sim \operatorname{Bernoulli}\left(q_{i}\right) \\
\delta_{i} & \sim D_{1} \\
u & \sim D_{2}
\end{aligned}
$$

Again, we might know the timescale. For example, if we have bioturbation in a sediment it might add a temporal offset between primary deposition and final location, which is of the order of a hundred years. We could express this as:

Outlier_Model ("TSimple",N (0,100), 0,"t");

This is the default outlier model applied for non- ${ }^{14} \mathrm{C}$ measurements if no other is specified. In most cases, however, we do not know the scale of any such offsets and so a more general model is more appropriate: 
Outlier_Model ("General", T (5), U (0,4), "t");

This is the model the author would recommend for most purposes. It draws from a long-tailed distribution $\left(D_{1}\right.$ is $\left.T(5)\right)$ and so will not be too affected by the odd extreme outlier and the scale (determined by the analysis) can be anywhere between $10^{\circ}$ and $10^{4} \mathrm{yr}\left(D_{2}\right.$ is $\left.U(0,4)\right)$.

In some instances, we might wish to use a more specific model. For example, consider the case of charcoal samples. These are often discounted or used only as a terminus post quem. However, in reality we know rather more than this. In particular, many charcoal dates are likely to be only very slightly earlier than the date of deposition with a long tail of older dates from old wood or redeposited charcoal. Such a distribution is likely to be approximately exponential (as suggested by Nicholls and Jones 2001) but with an unknown time constant (longer than a year but shorter than a thousand years). This can all be put into an outlier model suitable for charcoal:

Outlier_Model ("Charcoal ", $\operatorname{Exp}(1,-10,0), \mathrm{U}(0,3), " \mathrm{t} ")$;

Here we only allow outliers to be older, so the exponential distribution is taken to run from -10 to 0 with a time constant of 1 . The shifts are then scaled by a common scaling factor that can lie anywhere between $10^{0}$ and $10^{3} \mathrm{yr}$. In the case of charcoal samples, we know that all samples are expected to be outliers (that is, all earlier than context) and so they should be given a prior outlier probability of 1 .

\section{EXAMPLES}

\section{Combination of Dates for Samples of the Same Age}

One situation where outlier detection can be useful is when you have a large number of ${ }^{14} \mathrm{C}$ dates all pertaining to 1 context but measured by different laboratories using different techniques. The congruity of such a set can be tested using the non-Bayesian $\chi^{2}$ test of Ward and Wilson (1978). However, what do you do if the test fails? Using outlier detection, you can downweight those measurements that disagree most with the others and also identify which these are. As an example, we will take ${ }^{14} \mathrm{C}$ dates from the important context $\mathrm{X}$ in Tell Qasile as reported in Boaretto et al. (2005) and Sharon et al. (2007). The measurement history is complicated and will not be discussed here. We will take 11 of the measurements reported for this context, all of which are supposed to be the same age (Table 2).

Table 2 Dates from Tell Qasile X; the prior probability for each measurement being an outlier has been set to 0.05 (or 5\%); the analysis output provides posterior probabilities for each measurement being an outlier.

\begin{tabular}{llllll}
\hline Ref. & Lab ref. & Date & \pm & Prior & Posterior \\
\hline QS1 & T18161a, aa & 2818 & 26 & 0.05 & 0.08 \\
QS2 & RTT3932.3-6 & 2692 & 24 & 0.05 & 1.00 \\
QS3 & RTT3931.3-5 & 2911 & 26 & 0.05 & 0.62 \\
QS4 & LSC3931.1 & 2853 & 25 & 0.05 & 0.03 \\
QS5 & GrN27719 & 2895 & 25 & 0.05 & 0.33 \\
QS6 & RTT3853.1,3,4 & 2753 & 22 & 0.05 & 1.00 \\
QS7 & T3930 & 2800 & 25 & 0.05 & 0.33 \\
QS8 & T3933a, aa & 2882 & 28 & 0.05 & 0.10 \\
QS9 & GrA25535 & 2864 & 40 & 0.05 & 0.02 \\
QS10 & GrA25710 & 2818 & 38 & 0.05 & 0.04 \\
QS11 & GrA25768 & 2897 & 44 & 0.05 & 0.06 \\
\hline
\end{tabular}


The dates are all entered with a prior probability of being an outlier of 0.05 . The model outlined by Christen (2003) has been applied, but the treatment of the errors in the calibration curve is slightly different (see section "Statistical Details" below). This set of dates fails the $\chi^{2}$ test ( $d f=10, T=70$ cf. 18.3), but the advantage of this kind of analysis is that in a controversial case like this you do not need to make a qualitative assessment of which dates are most likely to be wrong. You can see from Table 2 that two of the dates are identified as being definite outliers (QS2 and QS6). One other date (QS3) is also more likely to be an outlier than not (Figures 1 and 2).

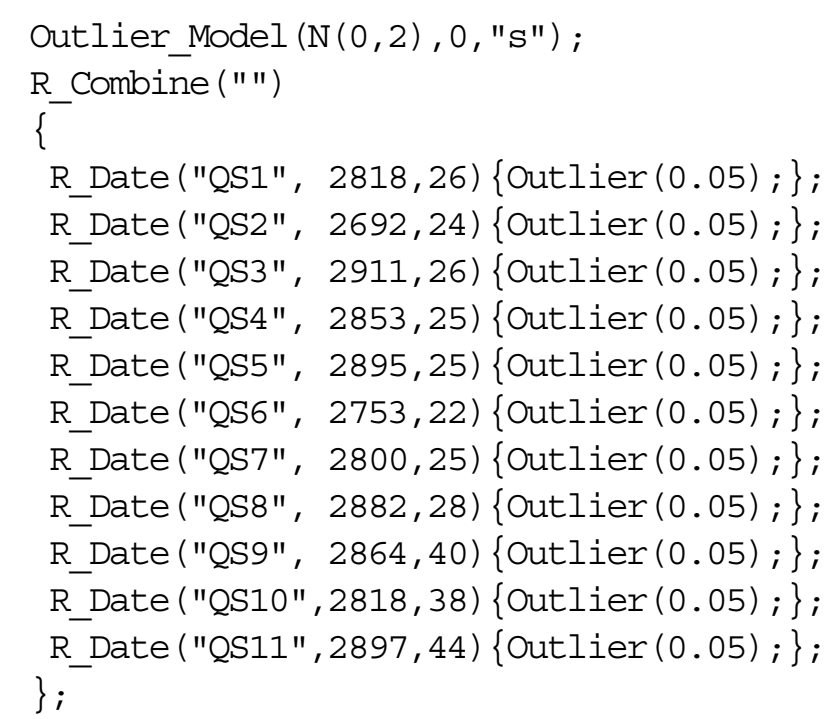

Figure 1 Model specification for outlier analysis of Tel Qasile dates

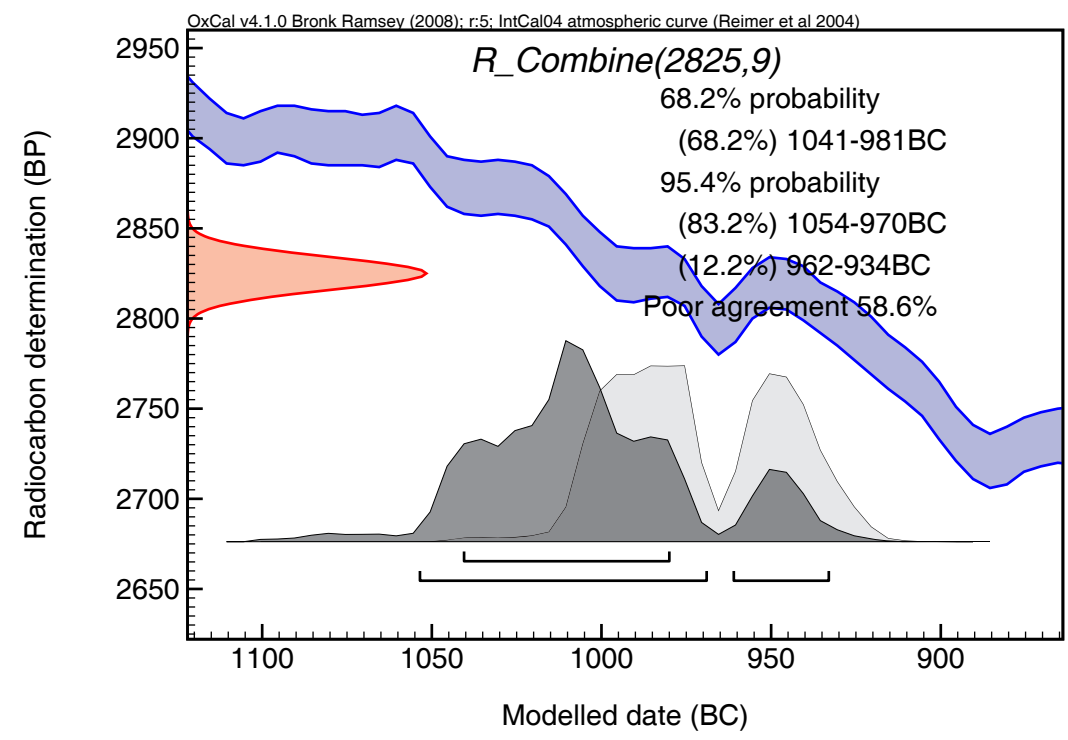

Figure 2 Combination of dates from tel Qasile X using the outlier analysis. The results of simple combination can be seen as an outline distribution in light gray; the results of the analysis are shown in darker gray and provide a significantly different age estimation. 
Of course, what any statistical analysis cannot do is identify the reasons why there are outliers. It could be that some of the samples really are of a different age, that there are contaminants present in some of the samples, or that there is a measurement problem of some kind. Outlier analysis is useful, however, in identifying which samples are most likely to be significantly wrong and providing an objective estimate of the true age of the sample set.

\section{Temporal Outliers in a Sedimentary Sequence}

The next example we will turn to is the situation where you have a sedimentary sequence where some of the samples are out of context and therefore give the wrong age for their depth. Such a situation might arise where there is significant bioturbation. To illustrate this example, we can look at the simulation data set 4 shown in Figure 6 of Blockley et al. (2007). In this case, some of the data points had been deliberately offset from their expected values to simulate the effect of outliers. Without the use of outlier analysis, it is necessary to work through the sequence eliminating those samples that have very low agreement indices in order to get a consistent model. In particular, 1 or 2 of the points are so far out that the model will not run with them included.

However, it is possible to use the general temporal outlier model described above instead of such a laborious and subjective procedure. Figure 3 shows how such a model is specified and Figure 4 the results of such an analysis, using a model averaging approach.

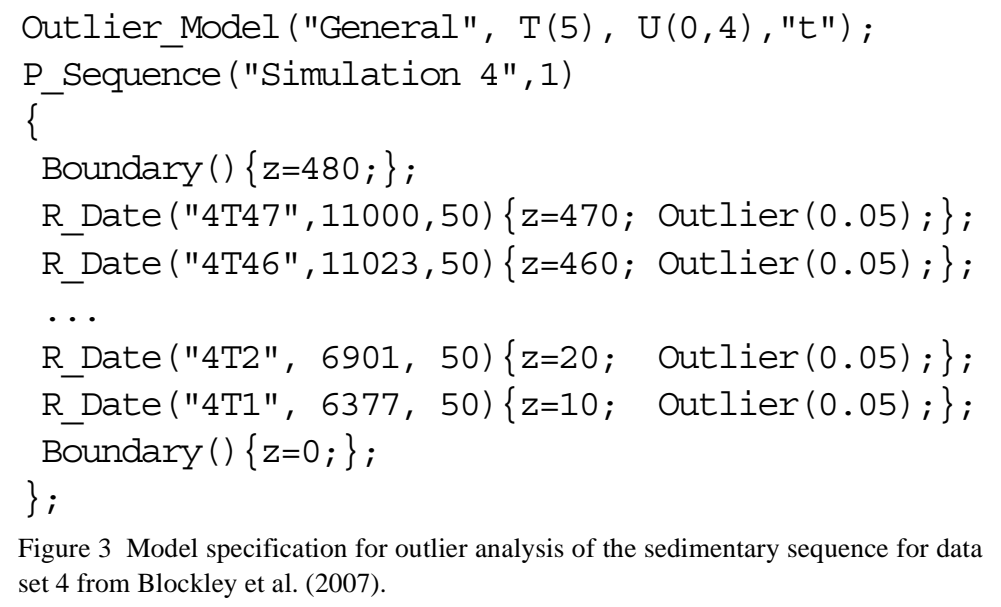

\section{Treatment of Charcoal Samples}

Here, we give a hypothetical example to show how this might work in practice. We have a single phase of occupation that is dated by some bone dates and a series of charcoal dates. The charcoal is not short-lived and so we assume that it must always be older than its context. Figure 5 shows how such a model is specified and Figure 6 shows the results of the analysis.

In this particular case, it can be deduced that the time-constant for residence of charcoal on the site lies in the range 10-100 yr (see Figure 6c). In this instance, the charcoal dates do add significantly to the model-many of the samples are no older than the bone dates and therefore provide important information on the date of the end of the phase and on its duration. 


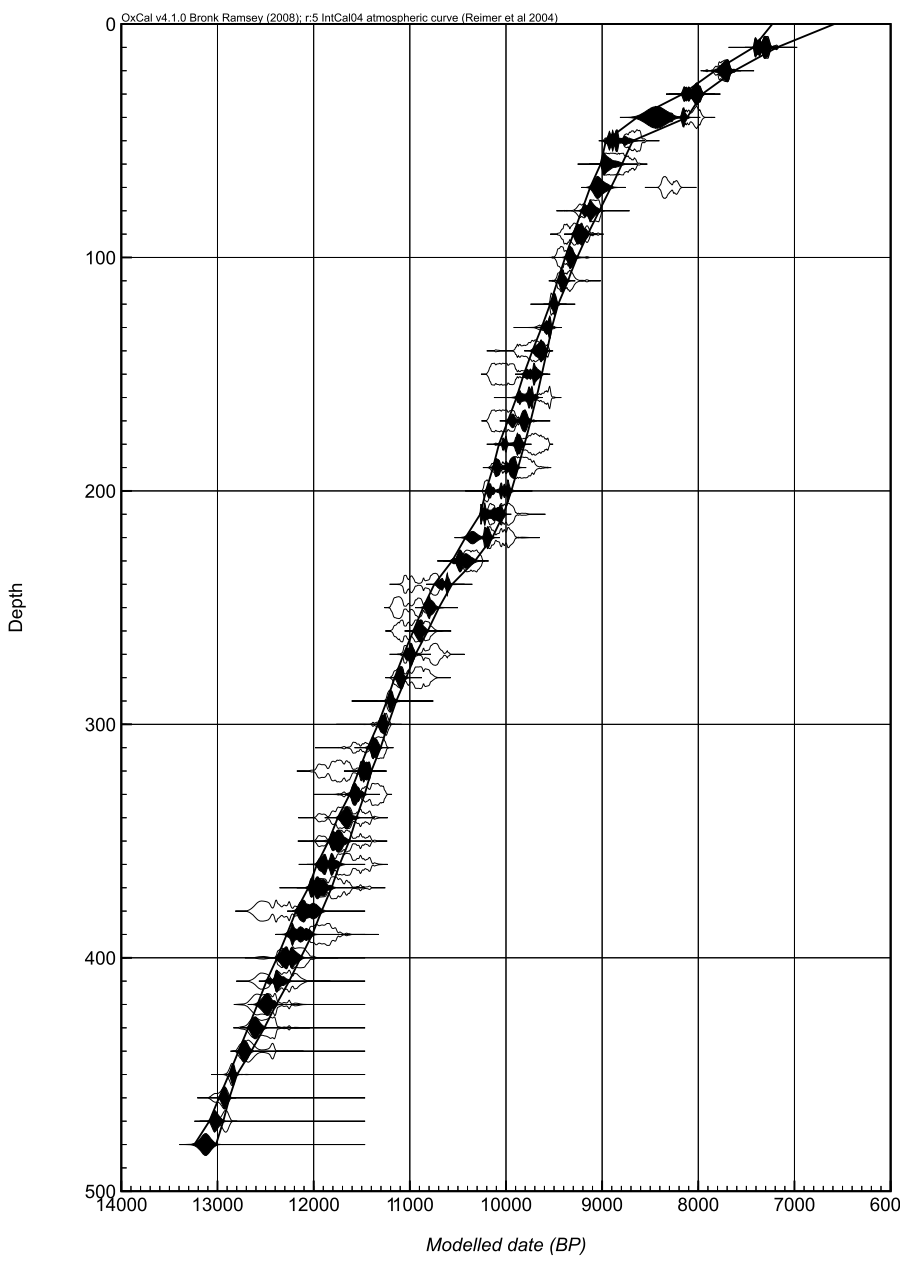

Figure 4 Age-depth model for data set 4 from Blockley et al. (2007) using outlier analysis. The results of simple calibration can be seen as an outline distribution with white fill; the results of the analysis are shown in black; you can see that some dates that are clearly outliers (such as the 7th from the top) are ignored in the analysis; this approach removes the need to weed out outliers manually before conducting such an analysis.

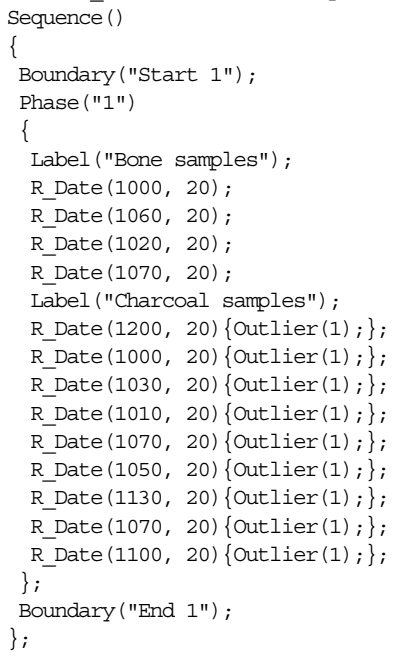

Figure 5 Model specification for a phase with bone and charcoal dates. Note that the Outlier command links to the last specified Outlier Model (in this case "Charcoal" if no name is specified. The command Outlier("Charcoal",1) could be used in each case instead. 


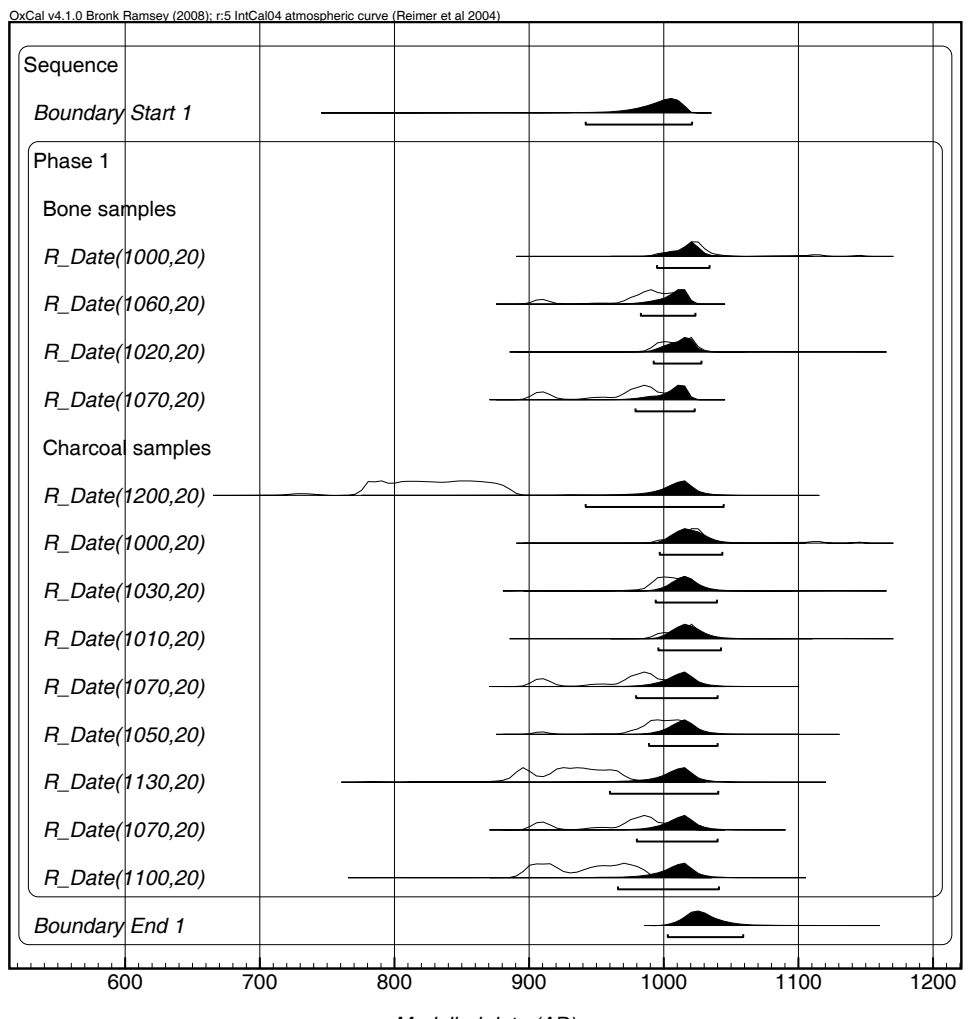

(a)

Modelled date $(A D)$

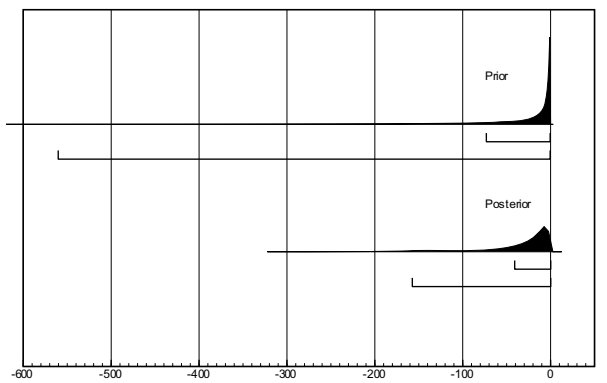

(b)

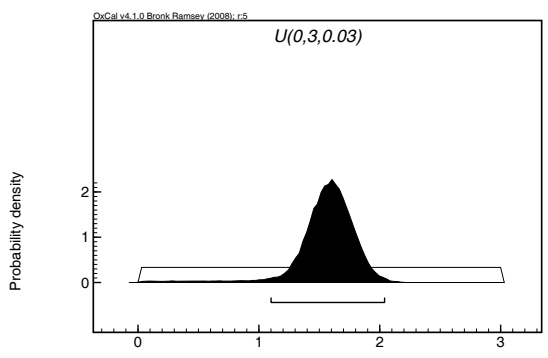

(c)

Figure 6 The results of the analysis of the model with charcoal dates. The charcoal dates act as a terminus ante quem for the end of the phase, but some are clearly much earlier than the start as you can see in the upper plot (a). The outline distributions show the simple calibrations and the black distributions show the estimate of the deposition dates of the samples $\left(t_{i}\right)$. In the lower left plot (b), you can see both the effective prior (from Equation 88) and the posterior distribution of the outlier offsets $\left(10^{u} \delta_{i}\right)$, which give an estimate for the charcoal ages on the site. In the lower right plot (c), the estimated timescale (in powers of 10) for charcoal residuality on the site (the posterior distribution for $u$ with the uniform prior shown in outline).

\section{Systematic Offsets Relative to the Calibration Curve}

As an example of such an application we can consider the data set of Imamura et al. (2007). They give data for 2 tree-ring sequences from Japan, one of which is a reference data set of known age (outer ring $\mathrm{AD} 350$ ) and another sample data set from a short 43-ring sequence that has been dendrochronologically dated (outer ring AD 389). The standard D_sequence analysis can be carried out 
for both series, but, although the reference series correctly dates to AD 335-357 (95.4\% probability), the other sample has a bimodal distribution with ranges AD 295-309 (86\%) or AD 373-387 (9\%) just missing the true value. If we now reanalyze the same 2 series together with a systematic $\Delta \mathrm{R}$ value of $0 \pm 10$, the first still dates to a range $\mathrm{AD} 334-361$ (95.4\%), consistent with the correct value. The other still has a bimodal distribution, but the ranges are AD 295-317 (47.5\%) and AD 370-395 (47.9\%), which is now in good agreement with the true value. The comparison between the 2 analyses can be seen in Figure 7. The reason that this works is that the analysis is able to make use of the fact that both series fit to the calibration curve better with a small systematic offset relative to the calibration curve. We can also get information on the nature of this shift. Figure 7 shows the prior and posterior for the $\Delta \mathrm{R}$, showing, in the posterior, a bimodal distribution. The shift to positive $\Delta \mathrm{R}$ gives the "correct" fit whereas that to the left gives an equally good but "wrong" fit for the data.
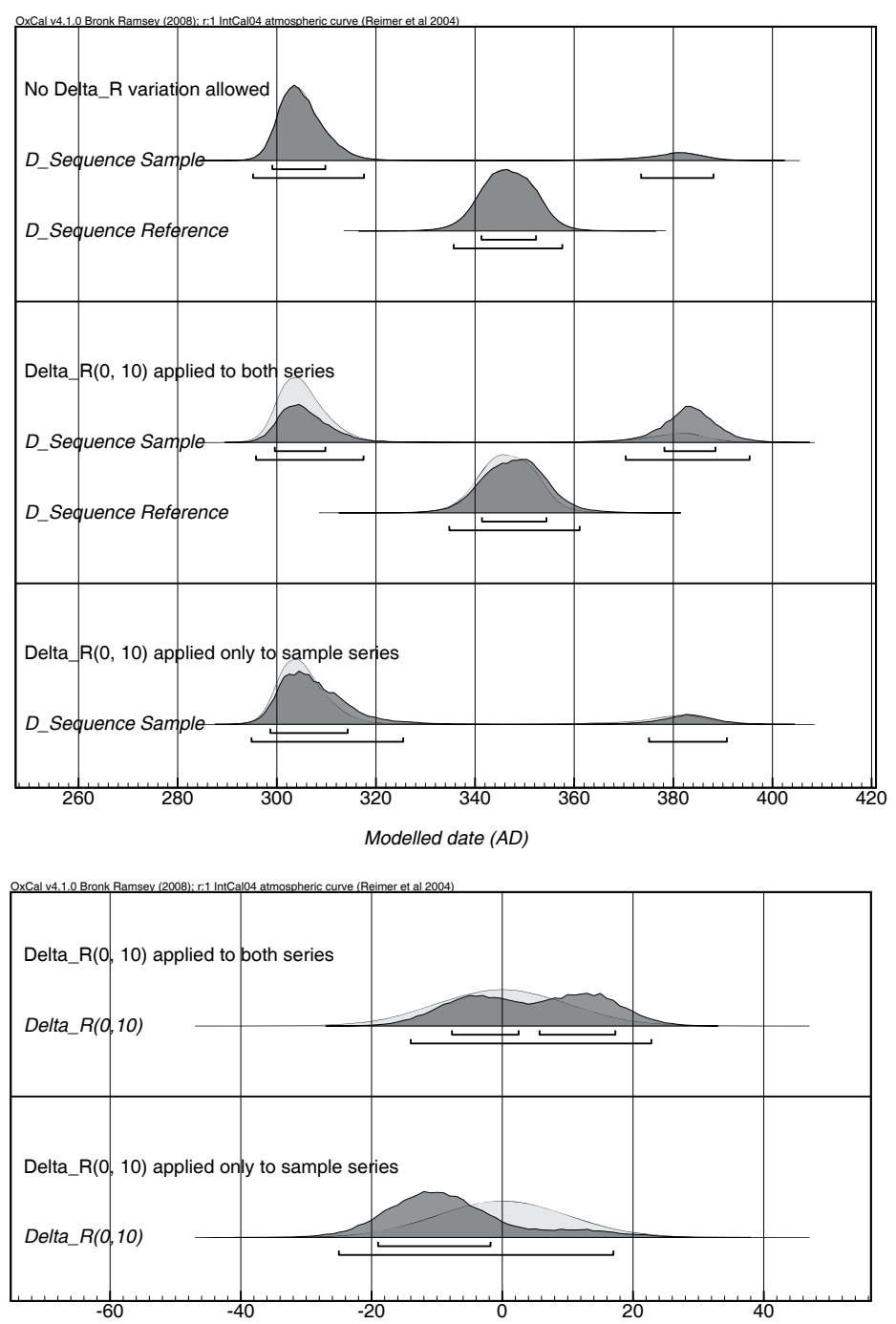

Figure 7 The results of the reanalysis of the data sets of Imamura et al. (2007). The upper figure shows the modeled end date for the sequences using the models described in the text (in dark gray) compared to those with no allowance for offsets (in light gray). The lower figure shows the posterior density estimates of the true reservoir offset (in dark gray) together with the priors (in light gray).

The analysis works well in this case because the reference data set is effectively able to inform the model about the offset—even though we have not used the calendar age in the analysis. If we use the 
same $\Delta \mathrm{R}$ value of $0 \pm 10$ and analyze the problematic sample series on its own, we still do better than with no allowance for $\Delta \mathrm{R}$ with a bimodal range of AD 294-325 (83.3\%) or AD 374-391 (12.1\%), which is just in agreement with the true value. There is still a substantially higher earlier peak since, even allowing for offsets, this series does match the earlier part of the curve slightly better (see Figure 7). What is clear from this analysis is that even a small allowance for systematic offsets can have a significant effect on the accuracy of the result. What the statistical analysis cannot tell us is whether the offset is due to differences in measurement or a true regional offset.

This kind of robustness test is very important even in cases where we do not expect outliers.

\section{STATISTICAL DETAILS}

In general, the treatment of outliers described here is embedded in more general Bayesian analysis. Bayes' theorem tells us that:

$$
p(\mathbf{t} \mid \mathbf{y}) \propto p(\mathbf{y} \mid \mathbf{t}) p(\mathbf{t})
$$

where $\mathbf{t}$ are the set of parameters and $\mathbf{y}$ the observations or measurements made. In this equation, $p(\mathbf{t})$ gives our prior knowledge about the parameters (this can include phase models, sequences, or deposition models as required). The part of the equation most important for this paper is the likelihood $p(\mathbf{y} \mid \mathbf{t})$, which is used to work out our posterior $p(\mathbf{t} \mid \mathbf{y})$. In many cases (when the data are conditionally independent), it is possible to factorize the likelihood into individual elements:

$$
p(\mathbf{y} \mid \mathbf{t})=\prod_{i} p\left(y_{i} \mid t_{i}\right)
$$

We use the approach of Abraham and Box (1978) as used by Christen (1994b) to deal with observations that are spurious in some way. In order to formalize this, we consider the form of the likelihood function $p\left(y_{i} \mid t_{i}\right)$. This will in general be some function of the observed variable(s) $y_{i}$ and the parameter(s) $t_{i}$ involved, where the age determination are indexed $i=1,2, \ldots, n$ :

$$
p\left(y_{i} \mid t_{i}\right)=l_{i}\left(y_{i}, t_{i}\right)
$$

In the case of ${ }^{14} \mathrm{C}$ dating, the observation consists of both the ${ }^{14} \mathrm{C}$ measurement $r_{i}$ and its uncertainty $s_{i}$. To use this for calibration, we also need to have a calibration curve that gives the expected ${ }^{14} \mathrm{C}$ concentration, $r(t)$ and the uncertainty $s(t)$ both as a function of calendar time. Using the usual error model, with an error of $\varepsilon_{i}$ for each measurement:

$$
\begin{gathered}
r_{i}=r\left(t_{i}\right)+\varepsilon_{i} \\
\varepsilon_{i} \sim N\left(0, s_{i}^{2}+\left(s\left(t_{i}\right)\right)^{2}\right)
\end{gathered}
$$

the ${ }^{14} \mathrm{C}$ likelihood function then becomes:

$$
p\left(r_{i} \mid t_{i}\right)=l_{i}\left(r_{i}, s_{i}, t_{i}\right) \propto \frac{\exp \left(-\frac{\left(r_{i}-r\left(t_{i}\right)\right)^{2}}{2\left(s_{i}^{2}+\left(s\left(t_{i}\right)\right)^{2}\right)}\right)}{\sqrt{s_{i}^{2}+\left(s\left(t_{i}\right)\right)^{2}}}
$$

In order to model the possibility of outliers, we introduce 2 more parameters for each observation. These parameters are $\phi_{i}$, which can take values 1 (if the observation is spurious and an outlier) or 0 (if the observation is correct), and $\delta_{i}$, which defines the offset in the observation if it is spurious. We 
define the priors for $\phi_{i}$ to be some predetermined value $q_{i}$ if it is 1 (an outlier) and $\left(1-q_{i}\right)$ if it is 0 (not an outlier):

$$
\phi_{i} \sim \operatorname{Bernoulli}\left(q_{i}\right)
$$

where the Bernoulli distribution is

$$
X \sim \operatorname{Bernoulli}(q) \Leftrightarrow \operatorname{Pr}(X=x)=q^{x}(1-q)^{1-x}
$$

the prior for $\delta_{i}$ also needs to be specified and normalized. We now need to consider the different forms of outliers.

\section{Outliers with Respect to the Time Parameter (t-type)\}}

In this case, what we are essentially modeling for is not a wrong measurement of a variable but a wrong interpretation in terms of the parameters of the model. In this case, we define the offset in terms of $t_{i}$, so that in the simplest case:

$$
p\left(y_{i} \mid t_{i}, \phi_{i}, \delta_{i}\right)=l_{i}\left(y_{i}, t_{i}+\phi_{i} \delta_{i}\right)
$$

Such a model can be specified in OxCal by specifying the prior distribution for $\delta_{i}$ and the outlier probability $q_{i}$. For example:

$$
\begin{aligned}
& \text { Outlier_Model ("TSimple",N(0,100),0,"t"); } \\
& \text { R_Date("OxA-12345",1423,23)\{Outlier(0.1,"TSimple"); ; }
\end{aligned}
$$

sets up the following priors and likelihood:

$$
\begin{aligned}
& r_{i}=r\left(t_{i}+\phi_{i} \delta_{i}\right)+\varepsilon_{i} \\
& \varepsilon_{i} \sim N\left(0, s_{i}^{2}+\left(s\left(t_{i}+\phi_{i} \delta_{i}\right)\right)^{2}\right) \\
& \delta_{i} \sim N\left(0,100^{2}\right) \\
& \phi_{i} \sim \operatorname{Bernoulli}(0.1) \\
& p\left(r_{i} \mid t_{i}, \phi_{i}, \delta_{i}\right)=l_{i}\left(r_{i}, s_{i}, t_{i}+\phi_{i} \delta_{i}\right) \\
& \propto \frac{\exp \left(-\frac{\left(r_{i}-r\left(t_{i}+\phi_{i} \delta_{i}\right)\right)^{2}}{2\left(s_{i}^{2}+\left(s\left(t_{i}+\phi_{i} \delta_{i}\right)\right)^{2}\right)}\right)}{\sqrt{s_{i}^{2}+\left(s\left(t_{i}+\phi_{i} \delta_{i}\right)\right)^{2}}}
\end{aligned}
$$

This is the default outlier model in OxCal for everything other than ${ }^{14} \mathrm{C}$ dates. However, in many cases we would rather not specify the functional form of the prior for $\delta$ so definitely. For this reason, we introduce a further model parameter, $u$, which provides the scale for all of the outliers associated with the model. In this model, the likelihood becomes:

$$
p\left(y_{i} \mid t_{i}, \phi_{i}, \delta_{i}, u\right)=l_{i}\left(y_{i}, t_{i}+10^{u} \phi_{i} \delta_{i}\right)
$$

We now need to provide a prior for $u$ as well and this is given as another parameter in the OxCal model definition. In addition, it is better to use a longer-tailed distribution than a normal distribution, and the Student's $t$ distribution with about 5 degrees of freedom is probably most useful for this (Venables and Ripley 2002:121). The reason for using such a long-tailed distribution in this type of 
model is that under the processes leading to temporal outliers there are sometimes a few very extreme outliers and we do not wish the modeled outlier distribution to be too heavily dependent on these. Putting all of this together, the following is a reasonable general outlier model for chronological applications:

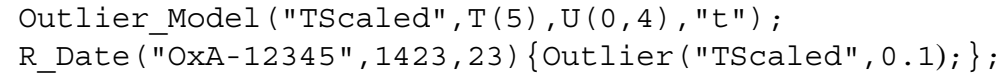

This sets up the following priors and likelihood:

$$
\begin{aligned}
r_{i} & =r\left(t_{i}+10^{u} \phi_{i} \delta_{i}\right)+\varepsilon_{i} \\
\varepsilon_{i} & \sim N\left(0, s_{i}^{2}+\left(s\left(t_{i}+10^{u} \phi_{i} \delta_{i}\right)\right)^{2}\right) \\
\delta_{i} & \sim T(5) \\
u & \sim U(0,4) \\
\phi_{i} & \sim \operatorname{Bernoulli}(0.1) \\
p\left(r_{i} \mid t_{i}, \phi_{i}, \delta_{i}, u\right) & =l_{i}\left(r_{i}, s_{i}, t_{i}+10^{u} \phi_{i} \delta_{i}\right)
\end{aligned}
$$

where the $T(v)$ is the Student's $t$ distribution with $v$ degrees of freedom:

$$
X \sim T(v) \Leftrightarrow \operatorname{Pr}(X=x) \propto\left(1+\frac{x^{2}}{v}\right)^{-\left(\frac{v+1}{2}\right)}
$$

The reason for choosing a log-uniform distribution for the scale of offsets is that many complex systems exhibit power-law dependency over a range of scales and the log-uniform distribution gives scale invariance. In practice, the outlier scale posterior is often approximately log-normally distributed and this is easily seen as a normal distribution in $u$ (as in Figure $6 \mathrm{c}$ ). The effective prior for the scaled offset $10^{u} \delta_{i}$ integrated over the range of values $0<u<4$ and with $\delta_{i} \sim T(5)$ is shown in Figure 8: this is a very long-tailed distribution. In most models, $u$ becomes fairly well constrained and the distribution becomes closer to the $T(5)$ distribution with an appropriate scale.

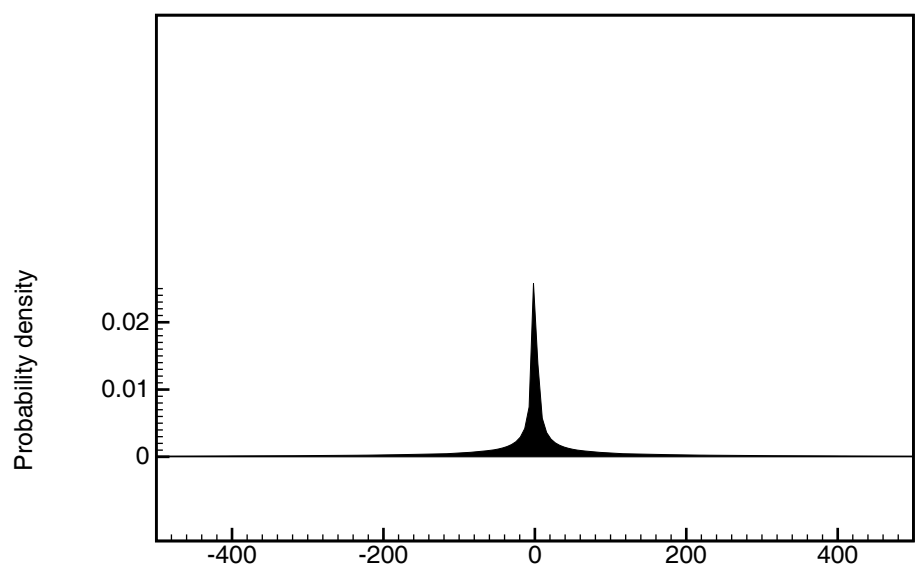

Figure 8 This shows the effective prior for the offsets in the General model with the full range of scales possible. The distributions is sharply peaked at zero (similar to the $T(5)$ distribution) but with very long shallow sloped tails. 
There are some dangers in having too many parameters in a model of this kind. In particular, if there are very few measurements, there may be confounding effects between the single $u$ parameter and the $\delta_{i}$ parameters. For this reason, with very small models it may be better to specify a fixed $u$. If you use the above model, you should look at the distribution for $u$ and check that the marginal posterior is somewhat constrained (see "Diagnosis" section below).

\section{Outliers with Underestimated Uncertainties (s-type)}

The construction of outliers with respect to the uncertainty quoted in the ${ }^{14} \mathrm{C}$ essentially follows the same pattern except that in this case the offset implied is in the ${ }^{14} \mathrm{C}$ measurement and not relative to the time parameter. This type of outlier obviously only makes sense in relation to ${ }^{14} \mathrm{C}$ measurements. For a simple implementation, we define the likelihood function to be:

$$
p\left(r_{i} \mid t_{i}, \phi_{i}, \delta_{i}\right)=l_{i}\left(r_{i}-\phi_{i} \delta_{i} s_{i}, s_{i}, t_{i}\right)
$$

This is essentially identical to the method proposed for generalized models in Christen (1994b) with the prior for the offset as defined in Christen (2003). The following model definition should reproduce the case where the assumed prior for $\delta_{i} \sim N\left(0,2^{2}\right)$ or for the offset in the ${ }^{14} \mathrm{C}$ date $\delta_{i} s_{i} \sim$ $N\left(0,\left(2 s_{i}\right)^{2}\right)$ :

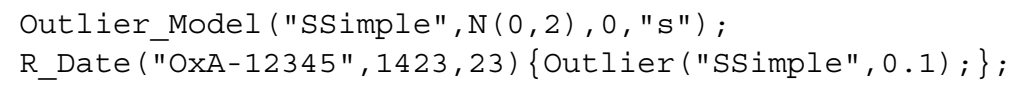

and sets up the following priors and likelihood:

$$
\begin{gathered}
r_{i}=r\left(t_{i}\right)+\varepsilon_{i}+\phi_{i} \delta_{i} s_{i} \\
\varepsilon_{i} \sim N\left(0, s_{i}^{2}+\left(s\left(t_{i}\right)\right)^{2}\right) \\
\delta_{i} \sim N\left(0,2^{2}\right) \\
\phi_{i} \sim \operatorname{Bernoulli}(0.1) \\
p\left(r_{i} \mid t_{i}, \phi_{i}, \delta_{i}\right)=l_{i}\left(r_{i}-\phi_{i} \delta_{i} s_{i}, s_{i}, t_{i}\right) \\
\propto \frac{\exp \left(-\frac{\left(r_{i}-\phi_{i} \delta_{i} s_{i}-r\left(t_{i}\right)\right)^{2}}{2\left(s_{i}^{2}+\left(s\left(t_{i}\right)\right)^{2}\right)}\right)}{\sqrt{s_{i}^{2}+\left(s\left(t_{i}\right)\right)^{2}}}
\end{gathered}
$$

This is the default outlier model applied by OxCal for ${ }^{14} \mathrm{C}$ dates if no other model is specified. It is equivalent to an increased variance $s_{i}$ when a measurement is identified as an outlier. The program is set up to use an optional scaling parameter for this type of offset too in which case the likelihood is given by:

$$
\begin{aligned}
r_{i} & =r\left(t_{i}\right)+\varepsilon_{i}+10^{u} \phi_{i} \delta_{i} s_{i} \\
u & \sim D_{2} \\
p\left(r_{i} \mid t_{i}, \phi_{i}, \delta_{i}, u\right) & =l_{i}\left(r_{i}-10^{u} \phi_{i} \delta_{i} s_{i}, s_{i}, t_{i}\right)
\end{aligned}
$$

where $D_{2}$ is specified as for the t-type model. However, the model as defined by Christen (1994b) is reasonable for most minor measurement problems and should probably be adopted as a standard 
model as it is for outlier detection of this sort. This model is implemented in BCal (Buck et al. 1999), Bwigg (Christen 2003), and Bpeat (Blaauw et al. 2003).

\section{Outliers in Radiocarbon Concentration (r-type)\}}

This implementation of the outlier model is essentially identical to that of the previous section, except that in this case we break the link with the original uncertainty in the measurement. The likelihood function is defined to be:

$$
p\left(r_{i} \mid t_{i}, \phi_{i}, \delta_{i}, u\right)=l_{i}\left(r_{i}-10^{u} \phi_{i} \delta_{i}, s_{i}, t_{i}\right)
$$

The definition of the model is then made in exactly the same way as for the t-type outliers:

Outlier_Model ("RScaled", T (5), U (0,4), "r") ;

R_Date ("OxA-12345",1423,23) \{Outlier("RScaled",0.1);\};

This sets up the following priors and likelihood:

$$
\begin{aligned}
& r_{i}=r\left(t_{i}\right)+\varepsilon_{i}+10^{u} \phi_{i} \delta_{i} \\
& \varepsilon_{i} \sim N\left(0, s_{i}^{2}+\left(s\left(t_{i}\right)\right)^{2}\right) \\
& \delta_{i} \sim T(5) \\
& u \sim U(0,4) \\
& \phi_{i} \sim \operatorname{Bernoulli}(0.1) \\
& p\left(r_{i} \mid t_{i}, \phi_{i}, \delta_{i}, u\right)=l_{i}\left(r_{i}-10^{u} \phi_{i} \delta_{i}, s_{i}, t_{i}\right) \\
& \\
& \propto \frac{\exp \left(-\frac{\left(r_{i}-10^{u} \phi_{i} \delta_{i}-r\left(t_{i}\right)\right)^{2}}{2\left(s_{i}^{2}+\left(s\left(t_{i}\right)\right)^{2}\right)}\right)}{\sqrt{s_{i}^{2}+\left(s\left(t_{i}\right)\right)^{2}}}
\end{aligned}
$$

\section{Offsets Relative to the Calibration Curve (d-type)}

This type of offset is essentially the same as the r-type outlier except in this case we assume that the offset is definite (the offset probability is 1) and the same offset applies to all (or a whole set of) ${ }^{14} \mathrm{C}$ measurements. Again, we introduce a new model parameter $d$, which defines the modeled offset between the calibration curve and the set of measurements. This is a single parameter that applies to all the dates (Jones and Nicholls 2001; Nicholls and Jones 2001). The likelihood for each ${ }^{14} \mathrm{C}$ measurement then becomes:

$$
p\left(r_{i} \mid t_{i}, d\right)=l_{i}\left(r_{i}-d, s_{i}, t_{i}\right) \propto \frac{\exp \left(-\frac{\left(r_{i}-\left(r\left(t_{i}\right)+d\right)\right)^{2}}{2\left(s_{i}^{2}+\left(s\left(t_{i}\right)\right)^{2}\right)}\right)}{\sqrt{s_{i}^{2}+\left(s\left(t_{i}\right)\right)^{2}}}
$$

In this equation, you can see why mathematically a bias can be treated in the same way as a reservoir offset. This kind of common offset is defined as a $\Delta R$ offset. In its more usual use, we have specific prior information for $\Delta \mathrm{R}$. However, it can be used more generally and if, for example, there is a pos- 
sible small but unknown offset between a set of measurements and the calibration curve, we might set up a model with:

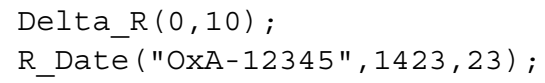

This will set up the following priors and likelihood:

$$
\begin{aligned}
r_{i} & =r\left(t_{i}\right)+\varepsilon_{i}+d \\
\varepsilon_{i} & \sim N\left(0, s_{i}^{2}+\left(s\left(t_{i}\right)\right)^{2}\right) \\
d & \sim N\left(0,10^{2}\right) \\
p\left(r_{i} \mid t_{i}, d\right)= & l_{i}\left(r_{i}-d, s_{i}, t_{i}\right) \\
\propto & \frac{\exp \left(-\frac{\left(r_{i}-d-r\left(t_{i}\right)\right)^{2}}{2\left(s_{i}^{2}+\left(s\left(t_{i}\right)\right)^{2}\right)}\right)}{\sqrt{s_{i}^{2}+\left(s\left(t_{i}\right)\right)^{2}}}
\end{aligned}
$$

Several Delta_R statements can be used in the same model, but the implementation in OxCal does not allow more than 1 such offset to apply to the same date.

\section{Sampling and Conditional Probabilities}

In all cases, OxCal uses a straightforward Metropolis-Hastings MCMC algorithm, so only relative probabilities are important. Each parameter of the outlier model is updated individually by sampling from the full conditionals. For all of the models outlined here, these are given by:

$$
\begin{aligned}
p\left(t_{i} \mid \mathbf{t}_{-i}, y_{i}, \phi_{i}, \delta_{i}, u, d\right) & \propto p(\mathbf{t}) p\left(y_{i} \mid t_{i}, \phi_{i}, \delta_{i}, u, d\right) \\
p\left(\phi_{i} \mid t_{i}, y_{i}, \delta_{i}, u, d\right) & \propto q_{i}^{\phi_{1}}\left(1-q_{i}\right)^{1-\phi_{1}} p\left(y_{i} \mid t_{i}, \phi_{i}, \delta_{i}, u, d\right) \\
p\left(\delta_{i} \mid t_{i}, y_{i}, \phi_{i}, u, d\right) & \propto p\left(\delta_{i}\right) p\left(y_{i} \mid t_{i}, \phi_{i}, \delta_{i}, u, d\right) \\
p(u \mid \mathbf{t}, \mathbf{y}, \mathbf{o}, \delta, d) & \propto p(u) \prod_{i} p\left(y_{1} \mid t_{1}, \phi_{i}, \delta_{1}, u, d\right) \\
p(d \mid \mathbf{t}, \mathbf{y}, \mathbf{o}, \delta, u) & \propto p(d) \prod_{i} p\left(y_{1} \mid t_{1}, \phi_{i}, \delta_{1}, u, d\right)
\end{aligned}
$$

\section{Radiocarbon Dates All Pertaining to One Event (s-type or r-type)}

As identified by Christen (1994b), the special case of combinations of ${ }^{14} \mathrm{C}$ dates all pertaining to one event needs to be treated slightly differently. The treatment presented there does not account for errors in the calibration curve and so a full treatment including these will be presented here.

Combination of ${ }^{14} \mathrm{C}$ dates is a 2 -stage process. The assumption is that all of the measurements relate to 1 calendar time and therefore all should correspond to the same original ${ }^{14} \mathrm{C}$ concentration, which we introduce as a parameter of the model $\rho_{c}$. Each measurement $\left(r_{i} \pm s_{i}\right)$ provides a likelihood function for this parameter: 


$$
p\left(r_{i} \mid \rho_{c}\right)=\frac{1}{s_{i} \sqrt{2 \pi}} \exp \left(-\left(r_{i}-\rho_{c}\right)^{2} /\left(2 s_{i}^{2}\right)\right)
$$

and thus for all of the measurements:

$$
p\left(\mathbf{r} \mid \rho_{c}\right) \propto \prod_{i} \exp \left(-\left(r_{i}-\rho_{c}\right)^{2} /\left(2 s_{i}^{2}\right)\right) / s_{i}
$$

Now for convenience we define:

$$
\begin{aligned}
& r_{c}=\left(\sum_{i} r_{i} / s_{i}^{2}\right) /\left(\sum 1 / s_{i}^{2}\right) \\
& s_{c}=\left(\sum_{i} 1 / s_{i}^{2}\right)^{-1 / 2} \\
& T=\sum_{i}\left(r_{i}-r_{c}\right)^{2} / s_{i}^{2} \\
& S=\prod_{i} \frac{1}{s_{i}}
\end{aligned}
$$

where $r_{c} \pm s_{c}$ is just the usual error-weighted combination of the ${ }^{14} \mathrm{C}$ dates (Ward and Wilson 1978). This allows us to factorize the likelihood as:

$$
p\left(\mathbf{r} \mid \rho_{c}\right) \propto S \exp (-T / 2) \exp \left(-\left(r_{c}-\rho_{c}\right)^{2} /\left(2 s_{c}^{2}\right)\right)
$$

This is just like the normally distributed likelihood for the ${ }^{14} \mathrm{C}$ ratio $\rho_{c}$ you would get for a single measurement but with a mean of $r_{c}$ and a standard deviation of $s_{c}$. The uncertainty in the calibration curve does not come in to the equation yet.

Now we need to consider the prior for the parameter $\rho_{c}$ in the model. As for a single calibration, this is given by:

$$
p\left(\rho_{c}, t_{c}\right) \propto \frac{1}{s(t)} \exp \left(-\left(\rho_{c}-r\left(t_{c}\right)\right)^{2} /(2 s(t))^{2}\right)
$$

since if we integrate over $\rho_{c}$ we get a constant value, independent of $t$. So given this, we can now integrate out the parameter $\rho_{c}$, which we do not need:

$$
\begin{aligned}
p\left(\mathbf{r} \mid \rho_{c}, t_{c}\right) & \left.\propto \frac{S}{s(t)} \exp (-T / 2) \exp \left(-\left(r_{c}-\rho_{c}\right)^{2} /\left(2 s_{c}^{2}\right)\right) \exp \left(-\left(\rho_{c}-r\left(t_{c}\right)\right)^{2} /(2 s(t))^{2}\right)\right) \\
p\left(\mathbf{r} \mid t_{c}\right) & \propto \int_{\rho_{c}=-\infty}^{\infty} p\left(\mathbf{r} \mid \rho_{c}, t_{c}\right) d \rho_{c} \\
& \propto S \exp (-T / 2) \frac{\exp \left(-\frac{\left(r_{c}-r\left(t_{c}\right)\right)^{2}}{2\left(s_{c}^{2}+\left(s\left(t_{c}\right)\right)^{2}\right)}\right)}{\sqrt{s_{c}^{2}+\left(s\left(t_{c}\right)\right)^{2}}}
\end{aligned}
$$


For the case where outliers are not considered, $T$ is a constant, and in any case $S$ is a constant.

There are a number of useful elements that emerge from this. First of all, $T$ as defined in Equation 73 is the test statistic described in Ward and Wilson (1978), which has a $\chi^{2}$ distribution with $n-1$ degrees of freedom (where $n$ is the number of combined ${ }^{14} \mathrm{C}$ dates). You can see that this is directly related (cf. Bronk Ramsey et al. 2001) to the probability of a particular set of ${ }^{14} \mathrm{C}$ determinations for any $\rho_{c}$ :

$$
p(\mathbf{r}) \propto \int_{\rho_{c}} \exp \left(-\left(r_{c}-\rho_{c}\right)^{2} /\left(2 s_{c}^{2}\right)\right) d \rho_{c} \propto \exp (-T / 2)
$$

We can also now expand this treatment to deal with outliers. We offset $r_{i}$ to $r_{i}^{\prime}$.

$$
r_{i}^{\prime}=\left\{\begin{array}{c}
r_{i}-\phi_{i} \delta_{i} s_{i} \text { for un-scaled s-type } \\
r_{i}-\phi_{i} \delta_{i} \text { for un-scaled r-type } \\
r_{i}-10^{u} \phi_{i} \delta_{i} s_{i} \text { for scaled r-type }
\end{array}\right.
$$

We repeat the same calculations to obtain $r_{c}{ }^{\prime}$ (this must be repeated for each iteration of the model).

$$
\begin{aligned}
p\left(t_{c} \mid \mathbf{r}, \mathbf{o}, \delta, d, u\right) & \propto p(\mathbf{t}) l_{c}\left(r_{c}{ }^{\prime}-d, s_{c}, t_{c}\right) \\
p\left(\phi_{i} \mid t_{c}, \mathbf{r}, \mathbf{o}_{-i}, \delta, d, u\right) & \propto q_{i}^{\phi_{i}}\left(1-q_{i}\right)^{1-\phi_{i}} \exp \left(-\left(r_{i}^{\prime}-r_{c}{ }^{\prime}\right)^{2} /\left(2 s_{i}^{2}\right)\right) l_{c}\left(r_{c}{ }^{\prime}-d, s_{c}, t_{c}\right) \\
p\left(\delta_{i} \mid t_{c}, \mathbf{r}, \mathbf{o}, \delta_{-i}, d, u\right) & \propto p\left(\delta_{i}\right) \exp \left(-\left(r_{i}{ }^{\prime}-r_{c}{ }^{\prime}\right)^{2} /\left(2 s_{i}{ }^{2}\right)\right) l_{c}\left(r_{c}{ }^{\prime}-d, s_{c}, t_{c}\right) \\
p\left(u \mid t_{c}, \mathbf{r}, \mathbf{o}, \delta, d\right) & \propto p(u) l_{c}\left(r_{c}{ }^{\prime}-d, s_{c}, t_{c}\right) \prod_{i} \exp \left(-\left(r_{i}{ }^{\prime}-r_{c}{ }^{\prime}\right)^{2} /\left(2 s_{i}^{2}\right)\right) \\
p\left(d \mid t_{c}, \mathbf{r}, \mathbf{o}, \delta, u\right) & \propto p(d) l_{c}\left(r_{c}{ }^{\prime}-d, s_{c}, t_{c}\right)
\end{aligned}
$$

\section{Charcoal Model}

It is worth looking in a little more detail at the outlier model for charcoal samples outlined above. This model covers a range of scales $u$ from 0 to 3 and so effective prior for a single $\delta$ would be:

$$
\begin{aligned}
p(\delta) & \propto \int_{u=0}^{3} \frac{\exp \left(\delta / 10^{u}\right)}{10^{u}} d u \\
& \propto \frac{e^{\delta}-e^{\delta / 1000}}{\delta}
\end{aligned}
$$

where $\delta$ is only allowed to be negative. In the suggested implementation, this is truncated at $-10,000$ $\mathrm{yr}$ and can therefore be normalized. It is a vague prior that is well behaved near $\delta=0$ and is plotted in Figure 6b. In practice, in any model the timescale $u$ is considerably constrained and so the distribution of outliers will be closer to a simple exponential. As above, it is important not to introduce too many different parameters into a model and in this case the model suggested is only suitable if there are many charcoal samples-if you only have 1 or 2 there will be confounding effects between the $u$ parameter and the $\delta_{i}$ parameters. 


\section{DIAGNOSIS AND ROBUSTNESS TESTING}

When using the models outlined here it is worth testing how robust the posteriors are to changes in the underlying models. This is one reason why it is important that all of the parameters of the models in OxCal are specified by the user and so can be altered to see if they affect the results. Robustness testing can easily be applied by trying different outlier models and prior outlier probabilities.

Using the scaling factor $10^{u}$ will allow the model averaging to cover a range of different scales and should achieve more robust results. This is not necessary when using the outlier methods only for outlier detection, but it can be important when the method is used for obtaining realistic posterior densities from the model average. One simple example demonstrates this fairly well: we consider the case of a simple sequence of dates, with one obvious outlier, analyzed under a number of different outlier models. The results of this are shown in Figure 9. Here you can see that in particular, use of the SSimple model (Figure 9a) actually puts quite strong constraints on measurements even if they are identified as outliers; this model is not very good for model averaging when the outliers are more extreme than the model intended. Using a longer-tailed Student's $t$ distribution (Figure 9b) helps with this, but allowing the scale to adapt to the data (Figure 9c) provides a better overall average result if the scale of outliers is not known in advance.
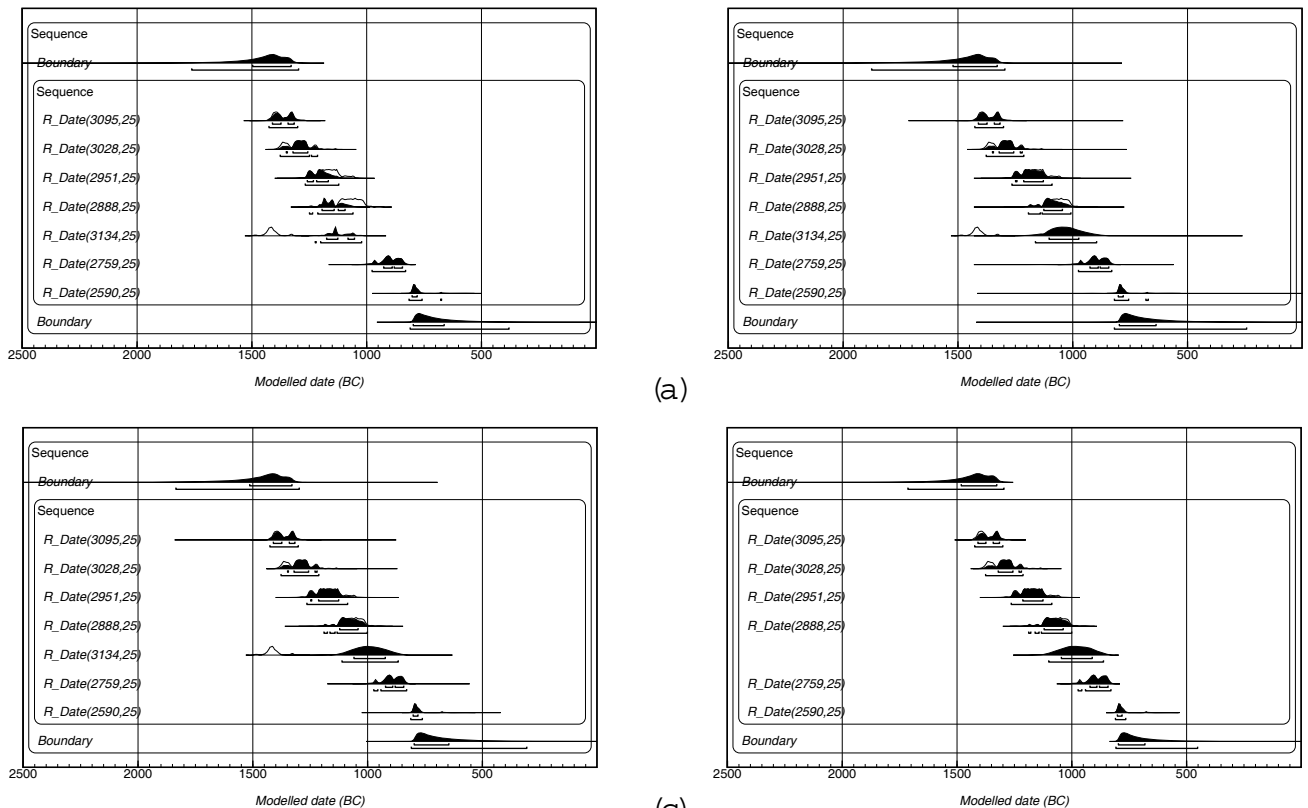

(a)

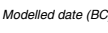

Figure 9 This show a simple sequence under a number of different outlier models: (a) uses the SSimple model as in Christen (2003); (b) uses a t-type outlier model with the offsets distributed as 100T(5) using Outlier_Model ("TFixed", T (5), 2, "t") ; (c) uses the General model as defined above with variable scaling; (d) manually removes the obvious outlier and replaces it with an undated event. All outlier models identify the fifth ${ }^{14} \mathrm{C}$ date as a definite outlier and can be used for outlier detection. However, given that the sample is an outlier, we would expect the modeled output to be similar to that shown in (d) where the date has been excluded: (a) shows that the very prescribed SSimple model pulls the outlier date strongly towards the measurement, even though it is an outlier; (b) which uses a longer-tailed $T(5)$ distribution is more realistic; but (c) gives a better overall model average for this situation since the dated event posteriors are very close to those shown in (d). 
The more complex scaled models do, however, come with the risk of confounding effects, where 1 parameter is played off against another and this is particularly true if for small models. It is possible for the user to check for model misbehavior by looking at 3 aspects of the model output: the convergence (see Bronk Ramsey 1995), the posterior distribution for the scaling parameter $u$, and the posterior outlier probabilities for $\phi_{i}$. A number of situations can arise:

- The convergence can be very slow. This is often associated with the scale of the offsets being hard to determine; in OxCal the model may never finish running at all if a satisfactory convergence is not achieved. In such circumstances, it may be necessary to use a simpler model.

- The distribution for $u$ may be poorly constrained and extend right up to the upper limit. This is normally the consequence of using the scaled model for a data set that is too small to support it; the results will still provide a model average over the specified scales, but it would usually be better to use a simpler model in such circumstances.

- The distribution for $u$ is well constrained at the upper limit but extends down to the lower limit. This is normally the consequence of having a data set where there may actually be no outliers at all and you will find that the outlier posterior probabilities for $\phi_{i}$ are very close to the priors $q_{i}$. In such a situation, there may be very small outliers that are undetectable and the model is reflecting this. This is not in itself a problem, but it does mean that the posteriors will be at least marginally affected by the lower limit set for the scale parameter $u$ and so a sensitivity test for this would be useful.

Ideally, the scaling parameter is well defined as in Figure $6 \mathrm{c}$ and the convergence is reasonably rapid (though it will always be slower than for a model without outliers).

There is one other consideration that has been taken into account in the implementation of these models and this is the special case where all of the measurements are outliers. In cases where this is the intention (as in the charcoal example above), this is not a problem. However, in most cases with the longer-tailed distributions suggested here there is such a range of possible solutions in such cases that this can lead to extremely slow convergence. For this reason, in cases where $q_{i} \neq 1$, and where there is more than 1 parameter tied to a particular outlier model, OxCal gives zero probability to the case where all measurements are outliers.

\section{CONCLUSIONS}

The approaches to dealing with outliers and offsets presented here are not only intended to provide detection of such offsets but also to provide good overall model averages that take into account a large range of different scenarios. For this reason, it is important, as in all Bayesian analysis, that the models used reflect the underlying mechanisms. This is why a number of different models are considered in this paper:

- s-type - where the ${ }^{14} \mathrm{C}$ measurement of a particular sample is wrong for some reason: these cases can be treated with shift outliers in the ${ }^{14} \mathrm{C}$ concentration as discussed in Christen (1994b, 2003).

- $\mathrm{r}$-type - where there are shifts between the ${ }^{14} \mathrm{C}$ concentration of the sample and its presumed reservoir, but where the measurement itself is accurate: in these cases a similar approach can be used but independent of the uncertainty in the measurement itself.

- d-type - where the ${ }^{14} \mathrm{C}$ measurements are biased relative to the calibration curve-either because of problems in the measurements or because of shifts in the ${ }^{14} \mathrm{C}$ ratio of the reservoir: these cases can be effectively treated in the same way as $\Delta \mathrm{R}$ offsets in marine ${ }^{14} \mathrm{C}$ calibration (Stuiver and Braziunas 1993; Jones and Nicholls 2001; Nicholls and Jones 2001). 
- $t$-type - where the sample measured might not relate to event being dated $(t)$ : in these cases outlier analysis using shifts in the calendar timescale can be used.

In the case of ${ }^{14} \mathrm{C}$ samples all relating to the same event, Ward and Wilson (1978) provide a useful test of whether measurements are all compatible. However, outlier analysis (s-type or r-type, depending on whether the problems are likely to relate to the measurement of the ${ }^{14} \mathrm{C}$ content of the sample) can be very useful in identifying which measurements are likely to be the outliers and giving a more objective assessment of the true age than manual rejection.

More generally where samples might or might not be outliers (as is usually the case), the methods outlined here allow an average of all of the possible combinations of rejection and acceptance of measurements to be averaged over, taking into account the posterior probabilities for such outliers. This model averaging approach is much more practical with large data sets than trying many different models each with different dates rejected. It is also more robust than selection of the outliers individually on the basis of agreement indices or outlier posterior probabilities and then just analyzing 1 model.

The implementation of all of these techniques in OxCal $\mathrm{v} 4.1$ has been presented so that researchers can apply them to their own projects. The tools provided are very flexible, but it should be stressed that it is probably best not to make any one model more complicated than it needs to be. For simple situations, with minor offsets, the original approach taken in Christen (2003) is likely to be sufficient for outlier detection. In larger models where displacement from context is often the main issue, the general t-type model should provide a good solution. In other cases, charcoal dates may need a more specific model. It is unlikely, however, that much will be gained by applying several different outlier models together, unless there are very good reasons for doing so.

The methods outlined here, if used in the right way, should start to address some of the problems associated with analyzing large numbers of ${ }^{14} \mathrm{C}$ dates and help to deal with issues of over-precision, which can arise if outliers and offsets are not considered at all.

\section{ACKNOWLEDGMENTS}

First of all, I would like to acknowledge the important work of Andrés Christen who was the first to look at the Bayesian treatment of outliers in ${ }^{14} \mathrm{C}$ dates (Christen 1994a) and who has made very valuable suggestions in the preparation of this paper. Martin Jones and Geoff Nicholls also made an important contribution in being the first to point out the need to treat $\Delta \mathrm{R}$ offsets as a correlated parameter. Mike Dee and Richard Staff have also helped to test the implementation of the outlier models in OxCal and made many useful suggestions. Thanks are also due to Geoff Nicholls who made many useful suggestions during the editing of this paper.

The research behind this paper was conducted in support of a Leverhulme-funded project on "Synchronising absolute scientific dating and the Egyptian historical chronology" (F/08 662/A). It also builds on work to develop OxCal funded by English Heritage (3164 MAIN). Without support from these institutions, this work could not have been completed.

\section{REFERENCES}

Abraham B, Box GEP. 1978. Linear models and spurious observations. Applied Statistics 27(2):131-8.

Blaauw M, Heuvelink GBM, Mauquoy D, van der Plicht J, van Geel B. 2003. A numerical approach to ${ }^{14} \mathrm{C}$ wiggle-match dating of organic deposits: best fits and confidence intervals. Quaternary Science Reviews
22(14):1485-500.

Blockley S, Blaauw M, Bronk Ramsey C, van der Plicht J. 2007. Building and testing age models for radiocarbon dates in Lateglacial and Early Holocene sediments. Quaternary Science Reviews 26(15-16):191526. 
Boaretto E, Jull AJT, Gilboa A, Sharon I. 2005. Dating the Iron Age I/II transition in Israel: first intercomparison results. Radiocarbon 47(1):39-55.

Bronk Ramsey C. 1995. Radiocarbon calibration and analysis of stratigraphy: the OxCal program. Radiocarbon 37(2):425-30.

Bronk Ramsey C. 2001. Development of the radiocarbon calibration program. Radiocarbon 43(2A):355-63.

Bronk Ramsey C. 2008. Deposition models for chronological records. Quaternary Science Reviews 27(1-2): 42-60.

Bronk Ramsey C, van der Plicht J, Weninger B. 2001. 'Wiggle matching' radiocarbon dates. Radiocarbon 43(2A):381-9.

Buck CE, Christen JA, James GN. 1999. BCal: an on-line Bayesian radiocarbon calibration tool. Internet Archaeology 7. Available at http://intarch.ac.uk/journal/ issue7/buck_index.html.

Christen JA. 1994a. Bayesian interpretation of radiocarbon results [PhD dissertation]. University of Nottingham.

Christen JA. 1994b. Summarizing a set of radiocarbon determinations: a robust approach. Applied StatisticsJournal of the Royal Statistical Society Series C 43(3): 489-503.
Christen JA. 2003. Bwigg: an internet facility for Bayesian radiocarbon wiggle matching. Internet Archaeology 7 . Available at http:/intarch.ac.uk/journal/issue13/ christe_index.html.

Imamura M, Ozaki H, Mitsutani T, Niu E, Itoh S. 2007. Radiocarbon wiggle-matching of Japanese historical materials with a possible systematic age offset. Radiocarbon 49(2):331-7.

Jones M, Nicholls G. 2001. Reservoir offset models for radiocarbon calibration. Radiocarbon 43(1):119-24.

Nicholls G, Jones M. 2001. Radiocarbon dating with temporal order constraints. Applied Statistics- Journal of the Royal Statistical Society Series C 50(4):503-21.

Sharon I, Gilboa A, Jull AJT, Boaretto E. 2007. Report on the first stage of the Iron Age Dating Project in Israel: supporting a Low Chronology. Radiocarbon 49(1): 146.

Stuiver M, Braziunas TF. 1993. Modeling atmospheric ${ }^{14} \mathrm{C}$ influences and ${ }^{14} \mathrm{C}$ ages of marine samples to 10,000 BC. Radiocarbon 35(1):137-89.

Venables WN, Ripley BD. 2002. Modern Applied Statistics. 4th edition. New York: Springer-Verlag.

Ward GK, Wilson SR. 1978. Procedures for comparing and combining radiocarbon age determinations: a critique. Archaeometry 20(1):19-31. 